\title{
Prescribing Patterns of Drugs in Pediatrics Outpatient Department in Tertiary Care Hospital
}

\author{
Surender Kagitapu ${ }^{1}$, Alekhya Nune ${ }^{2}$, Hemanth Devulapally ${ }^{2}$, Nagesh Adla ${ }^{2}$ \\ ${ }^{I}$ Department of Pediatrics, Kakatiya Medical College, Mahathma Gandhi Memorial Hospital, Warangal, \\ Telangana, India, 506002. \\ ${ }^{2}$ Department of Clinical Pharmacy, Vaagdevi College of Pharmacy, Kakatiya University, Hanamkonda, \\ Warangal, Telangana, India-506002
}

\begin{abstract}
Prescribing pattern studies are powerful exploratory tools to ascertain the role of drugs in society. In a tertiary care centre, prescribing is expected to be judicious, appropriate, safe, effective and economical. The ultimate goal is to achieve rational and effective medical care, particularly in the economically developing countries. An observational and prospective study was carried out for three months from June to August 2016. The study site was Paediatric out Patient Departments (OPD) of the Mahatma Gandhi Memorial Hospital, Warangal, Telangana. A total number of 18000 prescriptions were selected. The information such as age, sex and body weight was recorded. A total of 18000 prescriptions were analysed. The male patients were 9744 (54.13\%) and female patients were $8256(45.86 \%)$. Majority of the paediatric patients were suffering from respiratory tract infection followed by Diarrhea, Viral pyrexia, Epilepsy and Folliculitis. The number of drugs per counter was found to be with a minimum of 2 and maximum of 5 drugs. A total of 37468 drugs were prescribed and most frequently prescribed drug class was Paracetamol followed by Chlorphenaramine maleate, Oral rehydration salt, Amoxicillin, Sporolac, Zinc, Sodium valproate of total prescription.
\end{abstract}

Keywords: Pediatrics, respiratory tract infection, Paracetamol

\section{Introduction}

Prescribing pattern studies are powerful exploratory tools to ascertain the role of drugs in society. In a tertiary care centre, prescribing is expected to be judicious, appropriate, safe, effective and economical ${ }^{[1]}$. The ultimate goal is to achieve rational and effective medical care, particularly in the economically developing countries. This study is planned to analyze the prescribing pattern in pediatric outpatients attending at a tertiary care hospital. Paediatrics is the branch of medicine dealing with the development, diseases and disorders of children below 12 years. Medical treatment of a paediatric patient is based upon provisional and final diagnosis and optimum course of therapy, which involves a medication regimen. ${ }^{[2]}$ Infants and children are especially vulnerable to contact illnesses and to the harmful effect of drugs due to differences in pharmacodynamics and pharmacokinetics. ${ }^{[3]}$ Drug use in pediatrics is not extensively researched and the range of licensed drug in appropriate dosage form is limited when compared to adult medicine. ${ }^{[4]}$ Drug prescribing studies in pediatric patients have been limited when compared to Epidemiological evaluation of medicine use in elderly. The need for the safe and effective drugs for use in sick neonates, infants, children and adolescents requires the establishment of thoughtful drug therapy strategies. ${ }^{[5]}$ The study of prescribing patterns seeks to monitor, evaluate and if necessary suggest modifications in prescribing practices to make medical care rational and effective. ${ }^{[6]}$ The assessment of drug utilization is important for clinical, educational and economical reasons and study focuses on effective medical treatment of pediatric patients with accurate diagnosis and selecting the proper drug regimens, avoiding unnecessary use of antibiotics and minimizes the prescription errors.

\section{Materials And Methods}

An observational and prospective study was carried out for six months from June to August 2016. The study site was Paediatric out Patient Departments (OPD) of the Mahatma Gandhi Memorial Hospital, Warangal, Telangana. A total number of 18000 prescriptions were selected. The information such as age, sex and body weight was recorded. The drug data such as name of the drug, dosage form, dosing frequency, duration, route of administration and diagnosis data were also noted. The data obtained \& the patient related parameters were computed using Graphpad Prism version 5. The results were presented as percentage and mean \pm Standard deviation (SD).

\section{Results}

A total of 18000 prescriptions were analysed. The male patients were $9744(54.13 \%)$ and female patients were $8256(45.86 \%)$. The distributions and proportions are as shown in Figure 1. Majority of the paediatric patients were suffering from respiratory tract infection followed by Diarrhea, Viral pyrexia, Epilepsy 
and Folliculitis. The profiles of basis of morbidity are as shown in Figure 2. The classes of drugs prescribed are as shown in Figure 3. The number of drugs per counter was found to be with a minimum of 2 and maximum of 5 drugs. A total of 37468 drugs were prescribed and most frequently prescribed drug class was Paracetamol followed by Chlorphenaramine maleate, Oral rehydration salt, Amoxicillin, Sporolac, Zinc, Sodium valproate of total prescription. The classes of drugs prescribed are as shown in Figure 3. Age distribution was shown in figure 4 and distribution of various dosage forms was shown in figure 5.

Figure 1: Distribution of Male and Female ratio

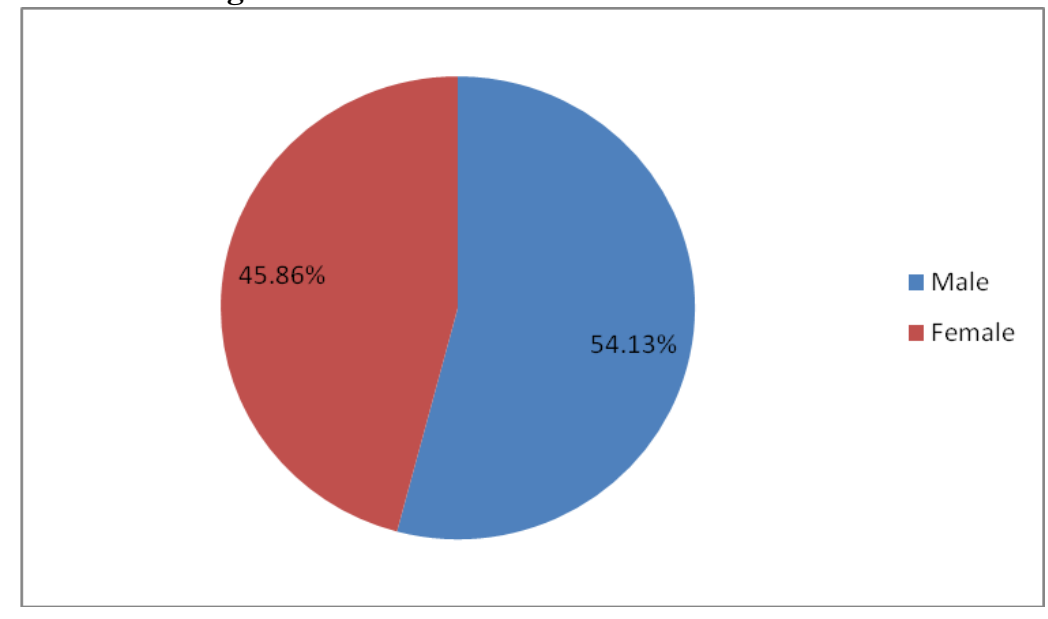

Figure 2: Distribution of percentages of various diseases

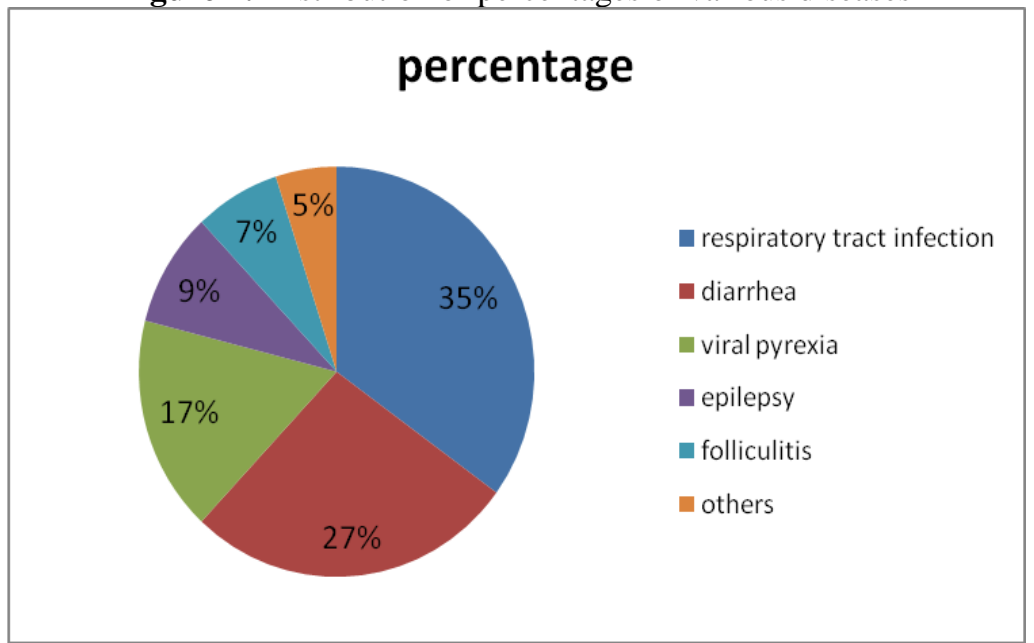

Figure 3: Distribution of percentages of various drugs

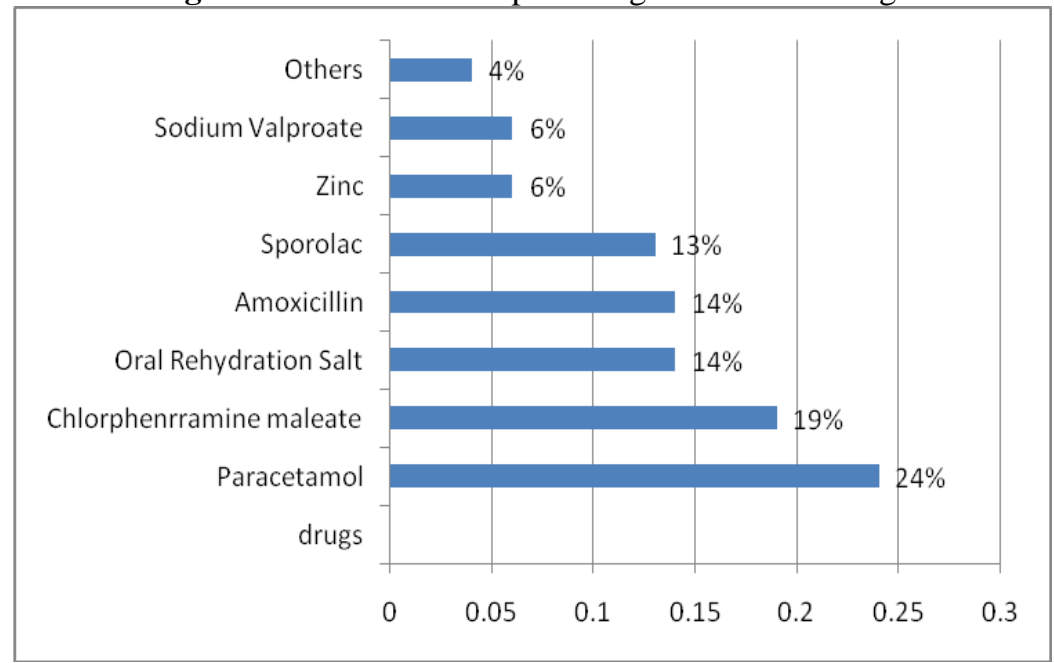


Figure 4: Distribution of Age wise distribution

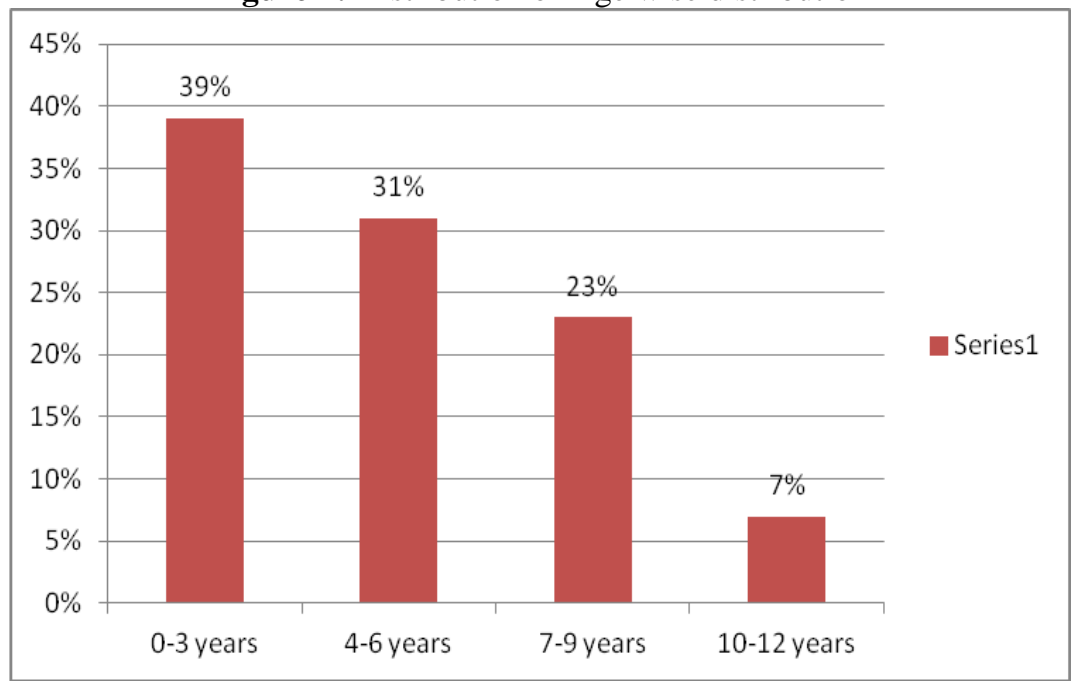

Figure 5: Distribution of various dosage forms

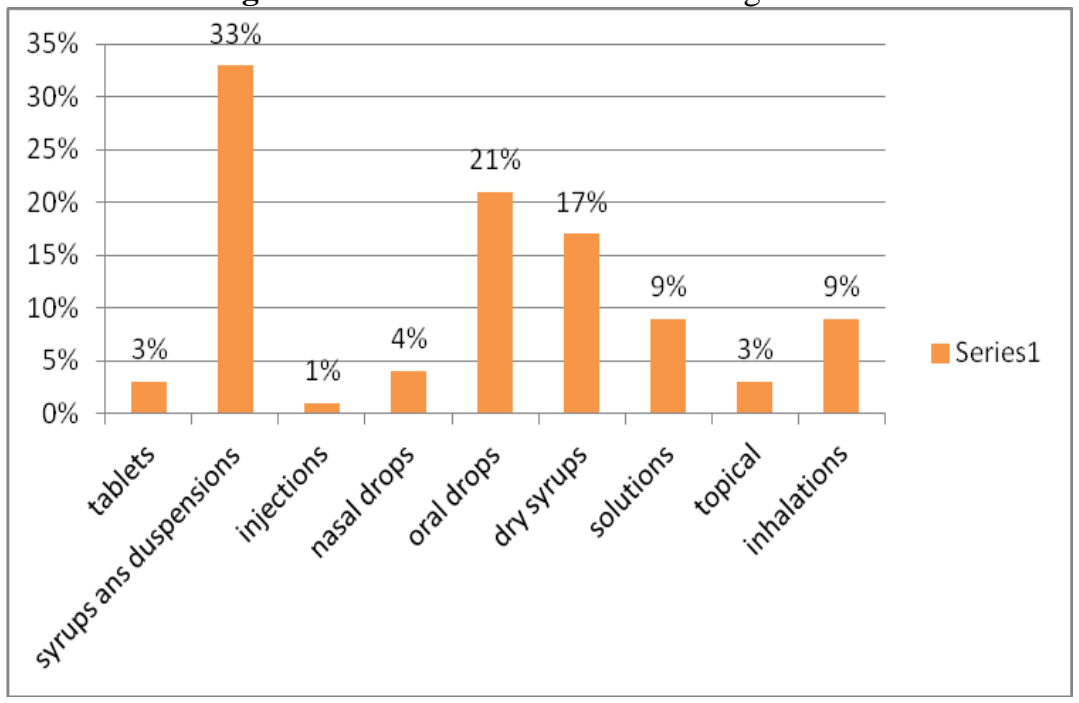

\section{Discussion}

Correct diagnosis of a disease \& its management with medicines constitute important aspect of patient care, which is more important in case of pediatric patient. The present study is based on data obtained from 18000 prescriptions. The male to female ratio reflected a higher number of male patients who are visiting clinic compared to female patients. About $35 \%$ patients were diagnosed with respiratory tract infection. $27 \%$ patients were found to be diagnosed with diarrhea. The most frequently prescribed drug class was Paracetamol. About 24\% patients have been prescribed with Paracetamol. Children differ from adults in their response to drugs. So, special care is needed in children and doses should always be calculated with care. $40 \%$ of patients were prescribed up to 2 drugs which was less than that in the study carried out 21 (i.e. $46.7 \%$ ) \& rest $60 \%$ were prescribed 3 to 5 drugs. ${ }^{[7]}$ It is comparatively less than the study done by Jason Hall 22 (i.e. 60.6\%). ${ }^{[8]} 2-3$ antibiotics were prescribed to $38.7 \%$ patients. In the study only $6 \%$ of antibiotics were given by intravenous injection which was quite opposite to the study done by Thomas G23 which indicated excessive use of injectables in many developing countries. ${ }^{[9]}$

\section{Conclusion}

This study provides prescribing patterns of drug use in the pediatrics outpatient department of a tertiary care teaching hospital. It has helped to identify rational prescribing patterns of drugs in pediatrics. Still, the clinical pharmacist must be considered to be an integral part of the multidisciplinary health care team. They should be involved in collection and presentation of prescribing data as part of clinical audit and also counselling of patients /care takers. Correct diagnosis of the disease and its management constitute important aspects of patient care which is even more important in case of pediatric patients. 


\section{References}

[1]. Ghai OP. Essential Pediatrics, published by interprint A-16, Narain all, new delhi, India. 4th edition. 1998.

[2]. Ghai OP, Paul VK. Rational drug therapy in pediatric practice. Indian paediatrics. 1988; 25(12): $1095-109$.

[3]. Summers RS, summers B. drug prescribing in paediatrics. Ann trop paediatrics. 1986; 6(2): 129-33.

[4]. Principi N. Control of antibiotic therapy in pediatric patients. Developmental pharmacology and therapeutics. 1981; 2(3): 145-55.

[5]. Schollenberg E, Albritton WL. Antibiotic misuse in a pediatric teaching hospital. Can Med Assoc J. 1980; $122(1): 49-52$.

[6]. Peter G, Micheal DR. Principles of Drug Therapy. In: Kliegman, Behrman, Jenson, Stanton, editors. Nelson Text book of Pediatrics. 18thedn. Vol 1. Philadelphia: Saunders Elsevier; 2007. p. 331-2.

[7]. Mirza Nazima Y, Desai Sagun, Ganguly Barna. Prescribing pattern in Out-patient department in Gujarat; Bangladesh. Journal of Pharmacology 2009; 4(1): 112-4.

[8]. Jason Hall BA. Pediatric prescribing in New Zealand. NZFP. 2002; 29 12): 14-8.

[9]. Tomson G. Drug utilization studies in Sri Lanka-Towards an understanding of medicine in society. Thesis, Karolinska Institute, Stokholm, Sweden; 1990; I : 1-5. 